\title{
Nonlinear collisionless damping of Weibel turbulence in relativistic blast waves
}

\author{
Martin Lemoine $\dagger$ \\ Institut d'Astrophysique de Paris, CNRS - UPMC, 98 bis boulevard Arago, F-75014 Paris, France
}

(Received 20 June 2014; revised 29 September 2014; accepted 30 September 2014; first published online 30 October 2014)

The Weibel/filamentation instability is known to play a key role in the physics of weakly magnetized collisionless shock waves. From the point of view of high energy astrophysics, this instability also plays a crucial role because its development in the shock precursor populates the downstream with a small-scale magneto-static turbulence which shapes the acceleration and radiative processes of suprathermal particles. The present work discusses the physics of the dissipation of this Weibelgenerated turbulence downstream of relativistic collisionless shock waves. It calculates explicitly the first-order nonlinear terms associated to the diffusive nature of the particle trajectories. These corrections are found to systematically increase the damping rate, assuming that the scattering length remains larger than the coherence length of the magnetic fluctuations. The relevance of such corrections is discussed in a broader astrophysical perspective, in particular regarding the physics of the external relativistic shock wave of a gamma-ray burst.

\section{Introduction}

The physics of collisionless shock waves has drawn wide interest, from pure theoretical plasma physics, starting with the pioneering work of Moiseev and Sagdeev (1963), to high energy astrophysics (e.g. Blandford and Eichler 1987), where it plays a key role in explaining most of the observed non-thermal spectra, and more recently, to laboratory high energy density physics, where collisionless shock waves are about to be produced through the interactions of laser beam-generated plasmas (e.g. Drake and Gregori 2012). At low magnetization - meaning that the unshocked plasma carries a magnetic field of small energy density compared to the shock kinetic energy - the physics of these collisionless shock waves is driven by the filamentation instability, also dubbed Weibel instability: this filamentation instability takes place in the shock precursor, where the incoming background plasma - as viewed in the reference frame in which the shock lies at rest - mixes with a population of shock-reflected or supra-thermal particles. This has been demonstrated by ab initio particle-in-cell (PIC) simulations, see e.g. Kato and Takabe (2008) for non-relativistic unmagnetized shock waves and Spitkovsky (2008a) for their relativistic counterparts, of direct interest to the present work. This filamentation instability and its various branches have consequently received a great deal of attention (see e.g. for relativistic shock waves Medvedev and Loeb 1999; Wiersma and Achterberg 2004; Lyubarsky and Eichler 2006; Achterberg and Wiersma 2007; Achterberg et al. 2007; Bret et al. 2010; Lemoine and Pelletier 2010, 2011; Rabinak et al. 2011; Shaisultanov et al. 2012).

$\dagger$ Email address for correspondence: lemoine@iap.fr 
Further simulations by Spitkovsky (2008b) have shown that, not only can Weibel/filamentation build up the electromagnetic barrier which gives rise to the shock transition through the isotropisation of the incoming background plasma, it also builds up the turbulence which is transmitted downstream of the shock, on plasma skin depth scales, and which provides the scattering centers for the Fermi acceleration process. Actually, the excitation of micro-turbulence - meaning a turbulence on scales smaller than the typical gyro-radius of accelerated particles - is a necessary condition for a proper relativistic Fermi process (Lemoine et al. 2006; Niemiec et al. 2006).

Additionally, Medvedev and Loeb (1999) have suggested that the filamentation mode is able to build up the turbulence in which the accelerated particles can lose their energy to secondary radiation through synchrotron (and possibly synchrotron selfCompton) processes $\uparrow$. In this unified picture, the filamentation instability that develops in the shock precursor would explain a variety of phenomena, from shock formation, to shock acceleration and even the non-thermal radiation from powerful astrophysical sources such as gamma-ray bursts. More particularly, the so-called gamma-ray burst afterglow radiation is attributed to the acceleration and (synchrotron) radiation of electrons at the external shock of the gamma-ray burst ultra-relativistic outflow, as it impinges on the very weakly magnetized circumburst medium. The phenomenological model of the afterglow provides a satisfactory description of most observed multiwavelength afterglow light curves, see e.g Piran (2004).

A notorious problem of the afterglow model remains to explain the origin of the magnetic field that permeates the blast, in which the electrons are assumed to radiate. Indeed, the turbulence which is generated through the Weibel/filamentation instability in the shock precursor and transmitted downstream is expected to decay rather quickly, on multiples of the skin depth scale (Gruzinov and Waxman 1999), while the time scales on which the electrons cool through synchrotron is of the order of $10^{8} \omega_{\mathrm{p}}^{-1}$ for typical external conditions. This remark has spurred many theoretical and numerical studies on energy transfer processes to long wavelengths (e.g. Medvedev et al. 2005; Katz et al. 2007), or alternative instabilities, which might re-amplify the magnetic field to a fraction of equipartition, from e.g. the interaction of the shock with an inhomogeneous medium (Sironi and Goodman 2007; Mizuno et al. 2014), or from a Rayleigh-Taylor instability at the contact discontinuity (Gruzinov 2000; Levinson 2009, 2010). How fast the Weibel-generated turbulence decays, thus, appears to be a key ingredient in shaping the light curves of relativistic blast waves.

Recent PIC simulations have addressed this dissipation issue. In PIC simulations of a relativistic collisionless pair shock up to time $5300 \omega_{\mathrm{p}}^{-1}$, Chang et al. (2008) have observed an isotropic, magneto-static turbulence downstream of the shock, which decays through phase mixing with a damping rate in rough agreement with the theoretical linear estimate. However, these authors point out that the linear calculation is ill-suited to describe the damping of the Weibel-generated turbulence in relativistic blast waves, since the trajectories of particles deviate from

$\dagger$ Strictly speaking, the relevant radiative processes in a microturbulence are jitter and jitter self-Compton, (see e.g. Medvedev et al. 2011; Kelner et al. 2013); however, close to the shock front of a relativistic collisionless shock wave, the Weibel-generated turbulence is of such strength that the jitter radiation boils down to the standard synchrotron spectrum in a coherent field of equivalent strength (Sironi and Spitkovsky 2009). Far from the shock, and in the presence of dissipation, jitter effects may in principle become significant, depending on how fast the field strength diminishes as the effective coherence length grows, see the discussion in Lemoine (2013). 
the ballistic regime. This remark has motivated the present study, which proposes to evaluate the first nonlinear corrections to the damping rate of such Weibelgenerated turbulence, accounting for the deviation of particle trajectories from straight lines.

The PIC simulations of Chang et al. (2008) have been essentially confirmed by the more extensive simulations of Keshet et al. (2009), although the latter authors observe that the acceleration of particles to progressively higher energies back reacts on the structure of the shock, and more importantly, on the power spectrum of the downstream turbulence, as suggested independently by Medvedev and Zakutnyaya (2009). Therefore, the former study concludes that present PIC simulations have not yet converged to a steady state. Since this longest PIC simulation $\left(\sim 10^{4} \omega_{\mathrm{p}}^{-1}\right)$ represents only a fraction of a percent of the dynamical timescale of the external shock wave of an actual gamma-ray burst, while particle acceleration and cooling is believed to take place on up to this latter timescale, this also means that theoretical extrapolation is needed to bridge the gap between these simulations and actual objects. Therefore, the damping rate, which depends on the power spectrum of the magnetic field, may well differ from that measured in these PIC simulations. This will be discussed in some detail further on.

In order to evaluate the nonlinear corrections to Landau damping, the present work calculates the nonlinear susceptibility in a magneto-static turbulence, following the Dupree-Weinstock description of resonance broadening (Dupree 1966; Weinstock 1969, 1970; Ben-Israel et al. 1975). This picture has been used in many studies to evaluate the saturation of instabilities through the back-reaction of particle diffusion in the grown turbulence, see e.g. (Dum and Dupree 1970; Bezzerides and Weinstock 1972; Weinstock 1972; Weinstock and Bezzerides 1973) and later works, e.g. Pokhotelov and Amariutei (2011) for the particular case of the Weibel temperature anisotropy. Here, it is used in a different context: downstream of the shock, the turbulence is magneto-static and isotropic, therefore the plasma is not subject to any instability, only to dissipation through phase mixing; the Dupree-Weinstock approach nevertheless allows to account for the influence of non-ballistic trajectories on the damping rate. Actually, it will be shown that a complete calculation of the first order nonlinear corrections is possible, since one can calculate explicitly the trajectory correlators in a magneto-static small-scale turbulence, following the method developed in Pelletier (1977) and Plotnikov et al. (2011).

The results obtained indicate a correction of order unity at the first nonlinear order. However, they also indicate that the correction systematically increases the damping rate, and that the magnitude of the correction versus the maximal wavenumber of the turbulence depends on the power spectrum of the magnetic field. These results are discussed in a broad context in Sec. 4. Section 2 provides the background for the calculation of the nonlinear damping rate, which is explicitly evaluated in Sec. 3. The trajectory correlators, which enter the calculation, are discussed in a separate Appendix B.

\section{Nonlinear damping of small-scale magnetostatic turbulence}

The initial set-up can be described as follows, in the rest frame of the (downstream) shocked plasma. Time $t=0$ corresponds to the time at which a given plasma element is advected through the shock towards downstream; while this plasma element has been crossing the shock precursor, it has been exposed to micro-instabilities which 
have built up a microturbulence to a level characterized by the parameter $\epsilon_{B}$ :

$$
\epsilon_{B}=\frac{\left\langle\delta B^{2}\right\rangle}{4 \pi\left(\gamma_{\mathrm{rel}}-1\right) n m c^{2}}
$$

with $m=m_{\mathrm{i}}$ for an electron-ion shock, $m=m_{e}$ for a pair shock; $n$ represents the particle density in the downstream plasma rest frame, and $\gamma_{\text {rel }}$ represents the Lorentz factor of the upstream plasma in the downstream rest frame; if $\gamma_{\mathrm{sh}} \equiv\left(1-\beta_{\mathrm{sh}}^{2}\right)^{-1 / 2}$ denotes the Lorentz factor of the shock front (and $\beta_{\mathrm{sh}}$ its velocity in units of $c$ ) relatively to the upstream plasma, $\gamma_{\text {rel }} \simeq \gamma_{\mathrm{sh}} / \sqrt{2}$ for a strong relativistic weakly magnetized shock (Blandford and McKee 1976). PIC simulations yield a value $\epsilon_{B} \sim$ $10^{-3}-10^{-2}$ immediately downstream of the shock (Keshet et al. 2009). The following calculations describe the microturbulence as aperiodic, i.e. $\mathfrak{R} \omega=0$, homogeneous and isotropic, as indicated by PIC simulations, see in particular Chang et al. (2008).

In this respect, the present set-up differs from that of Mart'yanov et al. (2008) and Kocharovsky et al. (2010) which derive stationary nonlinear and coherent magneto-static solutions to the Vlasov-Maxwell system in terms of inhomogeneous and anisotropic particle distribution functions. Such structures indeed emerge in the shock precursor in the nonlinear phase of the instability, as a balance between the anisotropy/inhomogeneity of the particle distribution functions and the magnetic forces. In the present case, the downstream particle distribution function is assumed homogeneous and isotropic, therefore the plasma is prone to collisionless damping. The homogeneity and isotropicity of the distribution function in the downstream plasma is a direct consequence of the shock transition, as clearly revealed by PIC simulations.

The present microturbulence also differs from the spontaneous turbulence associated to the thermal fluctuations of the plasma, as studied recently by Felten et al. (2013), Felten and Schlickeiser (2013a,b), Ruyer et al. (2013) or Yoon et al. (2014), since the present turbulence has been sourced in the shock precursor by the anisotropies of particle distribution functions.

Finally, the present work neglects any background magnetic field; in the case of the external shock wave of a gamma-ray burst, this is a very good approximation, since the magnetization parameter $\sigma \equiv B_{\mathrm{ISM} \mid \mathrm{d}}^{2} /\left[4 \pi\left(\gamma_{\mathrm{rel}}-1\right) n m c^{2}\right]$ expressed in terms of the downstream frame background field $B_{\mathrm{ISM} \mid \mathrm{d}}$ is very small compared to $\epsilon_{B}$ : $\sigma \sim 10^{-9}$ for typical interstellar conditions. Furthermore, the development of the relativistic Fermi acceleration process requires $\sigma \ll \epsilon_{B}^{2}$ (Pelletier et al. 2009; Lemoine and Pelletier 2010), i.e. a weakly magnetized shock wave in which the effects of the background magnetic field can be neglected.

\subsection{Damping of magneto-static turbulence}

Following Chang et al. (2008), one can use Poynting's theorem to derive the damping rate as a function of the transverse susceptibility. For random electric $\delta \boldsymbol{E}$ and magnetic fields $\boldsymbol{\delta} \boldsymbol{B}$ and random current density fluctuations $\boldsymbol{\delta} \boldsymbol{j}$ with zero spatial average, Maxwell equations imply

$$
\frac{1}{8 \pi c} \frac{\partial \delta B^{2}}{\partial t}+\frac{1}{8 \pi c} \frac{\partial \delta E^{2}}{\partial t}+\frac{1}{c} \boldsymbol{\delta} \boldsymbol{j} \cdot \boldsymbol{\delta} \boldsymbol{E}+\nabla \cdot(\boldsymbol{\delta} \boldsymbol{E} \times \boldsymbol{\delta} \boldsymbol{B})=0 .
$$

Then, taking the average over space, assuming homogeneous turbulence of strongly magnetic nature, which implies $\nabla \cdot\langle\boldsymbol{\delta} \boldsymbol{E} \times \boldsymbol{\delta} \boldsymbol{B}\rangle=0$ and $\delta E^{2} \ll \delta B^{2}$, one arrives at

$$
\frac{1}{8 \pi} \frac{\mathrm{d}\left\langle\delta B^{2}\right\rangle}{\mathrm{d} t}=-\langle\boldsymbol{\delta} \boldsymbol{j} \cdot \boldsymbol{\delta} \boldsymbol{E}\rangle
$$


or

$$
\begin{aligned}
\frac{1}{8 \pi} \frac{\mathrm{d}\left\langle\delta B^{2}\right\rangle}{\mathrm{d} t} & =-\frac{1}{2} \int \frac{\mathrm{d}^{3} k}{(2 \pi)^{3}} \frac{\mathrm{d} \omega}{2 \pi} \frac{\mathrm{d}^{3} k^{\prime}}{(2 \pi)^{3}} \frac{\mathrm{d} \omega^{\prime}}{2 \pi}\left\langle\boldsymbol{\delta} \boldsymbol{j}_{\boldsymbol{k} \boldsymbol{\omega}} \cdot \boldsymbol{\delta} \boldsymbol{E}_{\boldsymbol{k}^{\prime} \boldsymbol{\omega}^{\prime}}^{\star}+\boldsymbol{\delta} \boldsymbol{j}_{\boldsymbol{k} \omega}^{\star} \cdot \boldsymbol{\delta} \boldsymbol{E}_{\boldsymbol{k}^{\prime} \boldsymbol{\omega}^{\prime}}\right\rangle \\
& =\int \frac{\mathrm{d}^{3} k}{(2 \pi)^{3}} \frac{\mathrm{d} \omega}{2 \pi} \mathfrak{I}\left(\frac{1}{\omega \chi_{k \omega, \mathrm{T}}}\right) \mathscr{S}_{\delta j}(k, \omega) .
\end{aligned}
$$

The last equality uses the relation $\boldsymbol{\delta}_{\boldsymbol{k} \omega}=i \boldsymbol{\delta} \boldsymbol{j}_{\boldsymbol{k} \boldsymbol{\omega}} /\left(\omega \chi_{k \omega, \mathrm{T}}\right), \chi_{k \omega, \mathrm{T}}$ denoting the transverse susceptibility in $\omega-k$ space. It also introduces the power spectrum of current density fluctuations, through $\left\langle\delta j_{k \omega} \delta j_{k^{\prime} \omega^{\prime}}^{\star}\right\rangle=(2 \pi)^{4} \delta\left(\boldsymbol{k}-\boldsymbol{k}^{\prime}\right) \delta\left(\omega-\omega^{\prime}\right) \mathscr{S}_{\delta j}(k, \omega)$.

The transverse current density fluctuations are related to the transverse magnetic modes through (Felten et al. 2013)

$$
\boldsymbol{\delta}_{\boldsymbol{k} \omega}=\frac{i}{4 \pi} \boldsymbol{k} c \times \boldsymbol{\delta} \boldsymbol{B}_{\boldsymbol{k} \omega}\left[1+\frac{|\omega|^{4}}{(k c)^{4}}\right] .
$$

One can safely neglect the last term in the brackets since $|\omega|=\gamma_{k}$, the damping rate, and $\gamma_{k} \ll k c$ as demonstrated further on. Therefore, the power spectra of current fluctuations and magnetic turbulence are related through $\mathscr{S}_{\delta j}(k, \omega)=$ $(k c / 4 \pi)^{2} \mathscr{S}_{\delta B}(k, \omega)$ and for magneto-static turbulence, $\mathscr{S}_{\delta B}(k, \omega)=2 \pi \delta(\omega) \mathscr{S}_{\delta B}(k)$. One, thus, finally arrives at

$$
\frac{\mathrm{d}\left\langle\delta B^{2}\right\rangle}{\mathrm{d} t}=-2 \int \frac{\mathrm{d}^{3} k}{(2 \pi)^{3}} \gamma_{k} \mathscr{S}_{\delta B}(k)
$$

with damping rate in $k$-space ( $\gamma_{k}$ is counted as positive for effective damping):

$$
\gamma_{k}=-k^{2} c^{2} \mathfrak{I}\left(\frac{1}{4 \pi \omega \chi_{k \omega, \mathrm{T}}}\right)_{\omega \rightarrow 0} .
$$

Therefore, the bulk of the calculation consists in evaluating the nonlinear susceptibility. For reference, assuming ballistic trajectories and low frequencies $\omega \ll k c$, one has

$$
4 \pi \chi_{k \omega, \mathrm{T}} \simeq-i \frac{\pi}{4} \sum_{\alpha} \frac{4 \pi q_{\alpha}^{2}}{\omega k} \int \mathrm{d} p p^{2} \frac{\mathrm{d} \bar{f}_{\alpha}}{\mathrm{d} p} \Theta\left[1-\left(\frac{\omega}{k v}\right)^{2}\right]+\mathcal{O}\left(\omega^{0}\right),
$$

where $\bar{f}_{\alpha}(p)$ represents the homogeneous part of the distribution function of particles of species $\alpha$. For a Jüttner-Synge distribution:

$$
\bar{f}_{\alpha}(p)=\frac{n_{\alpha} \mu}{4 \pi m^{3} c^{3} K_{2}(\mu)} e^{-\mu \gamma}
$$

with $\mu=m c^{2} /(k T), \gamma=\left[1+p^{2} /(m c)^{2}\right]^{1 / 2}$ and $n_{\alpha}$ the density of particles, one finds as $\omega \rightarrow 0$

$$
4 \pi \chi_{k \omega, \mathrm{T}} \simeq i \frac{\pi}{4} \sum_{\alpha} \frac{\omega_{\mathrm{p}, \alpha}^{2}}{\omega k c} \frac{1}{K_{2}(\mu)}\left(\frac{2}{\mu}+\frac{2}{\mu^{2}}\right) e^{-\mu},
$$

in terms of the relativistic plasma frequency (squared) $\omega_{\mathrm{p}, \alpha}^{2}=4 \pi n_{\alpha} q_{\alpha}^{2} \mu / m$ which leads to the ultra-relativistic $(\mu \rightarrow 0)$ linear damping rate

$$
\gamma_{k} \simeq \frac{4}{\pi} \frac{k^{3} c^{3}}{\omega_{\mathrm{p}}^{2}}
$$

with $\omega_{\mathrm{p}}^{2}=\sum_{\alpha} \omega_{\mathrm{p}, \alpha}^{2}$ the relativistic plasma frequency of the global plasma. This result for the linear Landau damping rate in a ultra-relativistic plasma matches previous 
derivations, e.g. Bergman and Eliasson (2001), Chang et al. (2008) and Felten and Schlickeiser (2013b).

One can generalize very easily the above result to a power-law distribution of particles with index $s$ and minimum Lorentz factor $\gamma_{\min }$ :

$$
\bar{f}_{\alpha}(p)=\frac{n_{\alpha}|s-1|}{4 \pi m^{3} c^{3} \gamma_{\min }}\left(\frac{\gamma}{\gamma_{\min }}\right)^{-s-2} \Theta\left(\gamma-\gamma_{\min }\right)
$$

One then infers in the ultra-relativistic limit $\gamma_{\min } \gg 1$

$$
\gamma_{k}=\frac{4}{\pi} \frac{k^{3} c^{3}}{\omega_{\mathrm{p}}^{2}} \frac{s}{|(s+2)(s-1)|}
$$

The damping rate differs from the previous by a factor of order unity only. In the following, the calculation of the nonlinear damping rate will be carried out for this power-law distribution function, since it guarantees that there are no particle with Lorentz factor outside the range of application of the approximation used (see further below). Furthermore, one expects the distribution function in astrophysical blast waves to follow such a power-law to a good approximation; notably, Fermi acceleration at relativistic shock waves predicts a spectral index $s \simeq 2.3$ in the ultrarelativistic limit for isotropic scattering (e.g. Bednarz and Ostrowski 1998; Kirk et al. 2000; Achterberg et al. 2001; Lemoine and Pelletier 2003; Keshet and Waxman 2005).

\subsection{Nonlinear susceptibility}

The current density fluctuations, from which one can extract the susceptibility, are defined in terms of the fluctuating part of the distribution function, as:

$$
\boldsymbol{\delta} \boldsymbol{j}=\sum_{\alpha} q_{\alpha} \int \mathrm{d}^{3} r \boldsymbol{v} \delta f_{\alpha}(\boldsymbol{r}, \boldsymbol{p}, t)
$$

The full distribution function is written $f_{\alpha}(\boldsymbol{r}, \boldsymbol{p}, t)=\bar{f}_{\alpha}(\boldsymbol{p}, t)+\delta f_{\alpha}(\boldsymbol{r}, \boldsymbol{p}, t)$, with $\delta f_{\alpha}(\boldsymbol{r}, \boldsymbol{p}, t)$ the random inhomogeneous part and $\bar{f}_{\alpha}(\boldsymbol{p}, t)$ the spatial average. Following Weinstock $(1969,1970)$; Ben-Israel et al. (1975) this fluctuating part is given by the solution to the inhomogeneous part of the Boltzmann equation, and it can be written in terms of a propagator $\mathscr{U}_{\mathscr{A}}$ as:

$$
\delta f_{\alpha}(\boldsymbol{r}, \boldsymbol{p}, t)=\mathscr{U}_{\mathscr{A}}\left(t, t_{0}\right) \delta f_{\alpha}\left(\boldsymbol{r}, \boldsymbol{p}, t_{0}\right)-\int_{t_{0}}^{t} \mathrm{~d} \tau \mathscr{U}_{\mathscr{A}}(t, \tau) \boldsymbol{\delta} \mathscr{F}(\tau) \cdot \frac{\mathrm{d} \bar{f}_{\alpha}(\boldsymbol{p}, \tau)}{\mathrm{d} \boldsymbol{p}} .
$$

The random force operator is $\delta \mathscr{F}(\tau) \equiv q_{\alpha}[\boldsymbol{\delta} \boldsymbol{E}(\boldsymbol{r}, \tau)+\boldsymbol{v} \times \boldsymbol{\delta} \boldsymbol{B}(\boldsymbol{r}, \tau) / c]$. In the following, $\bar{f}_{\alpha}$ is assumed isotropic in $p$; then the term associated to the magnetic Lorentz force vanishes in the above expression.

The properties of $\mathscr{U}_{\mathscr{A}}$ are described in details in the above references and its relation to other propagators is discussed in Birmingham and Bornatici (1971). For the sake of completeness, their definitions are recalled in Appendix A.

In the following, the initial data will be written init. data out of brevity and clarity. Going over to Fourier variables,

$$
\begin{aligned}
\delta f_{\alpha \boldsymbol{k}}(\boldsymbol{p}, t)= & \text { init. data }-q_{\alpha} \int \mathrm{d}^{3} r \int \frac{\mathrm{d}^{3} k^{\prime}}{(2 \pi)^{3}} e^{-i \boldsymbol{k} \cdot \boldsymbol{r}} \\
& \times \int_{t_{0}}^{t} \mathrm{~d} \tau \mathscr{U}_{\mathscr{A}}(t, \tau) e^{i \boldsymbol{k}^{\prime} \cdot \boldsymbol{r}} \boldsymbol{\delta} \boldsymbol{E}_{\boldsymbol{k}^{\prime}}(\tau) \cdot \frac{\boldsymbol{v}}{v} \frac{\mathrm{d}}{\mathrm{d} p} \bar{f}_{\alpha}(p, t) .
\end{aligned}
$$


So far, the treatment has been exact; in particular, the separation of $f_{\alpha}(\boldsymbol{r}, \boldsymbol{p}, t)$ into an average and a random part does not imply any linearisation procedure. The main approximation of the present work is to approximate the full propagator $\mathscr{U}_{\mathscr{A}}$ by the average propagator $\overline{\mathscr{U}}$, which corresponds to the truncation to the first term in a series expansion in powers of the fields, see Appendix A, which summarizes the properties of these propagators, and see most notably Dupree (1966); Weinstock (1969, 1970); Birmingham and Bornatici (1971) and Ben-Israel et al. (1975). As recalled in Appendix A, higher order terms are suppressed relative to this first order correction by powers of $c \tau_{\mathrm{c}} / r_{\mathrm{g}}$, with $\tau_{\mathrm{c}}$ the correlation time of the electromagnetic fluctuations, $r_{\mathrm{g}}$ the typical gyroradius of the particles in the turbulence, defined with respect to $\left\langle\delta B^{2}\right\rangle^{1 / 2}$. The present work, thus, makes the explicit assumption that $r_{\mathrm{g}}>c \tau_{\mathrm{c}}$.

In relativistic blast waves, the typical Lorentz factor of a particle downstream of a relativistic shock wave of Lorentz factor $\gamma_{\mathrm{sh}}$ is $\gamma_{\mathrm{sh}}$ for a pair shock, or $\gamma_{\mathrm{sh}}$ (resp. $\gamma_{\mathrm{sh}} m_{\mathrm{i}} / m_{e}$ ) for the ion (resp. electron) population in an electronion shock (e.g. Spitkovsky 2008a,b). In the following, this Lorentz factor is denoted $\gamma_{\min }$. One then derives the typical ratio $r_{\mathrm{g}} / c \tau_{\mathrm{c}}$ for a particle of Lorentz factor $\gamma$ :

$$
\frac{r_{\mathrm{g}}}{c \tau_{\mathrm{c}}} \simeq \epsilon_{B}^{-1 / 2} \frac{k_{\max } c}{\omega_{\mathrm{p}}} \frac{\gamma}{\gamma_{\min }} .
$$

The typical scale of Weibel turbulence is $c \tau_{\mathrm{c}}=k_{\max }^{-1} \sim \mathscr{K} c / \omega_{\mathrm{p}}$ with $\mathscr{K} \simeq 10$ close to the shock front (Chang et al. 2008; Spitkovsky 2008a; Keshet et al. 2009; Sironi et al. 2013). Given that $\epsilon_{B} \lesssim 10^{-2}$, this indicates that typically, $r_{\mathrm{g}} \gtrsim c \tau_{\mathrm{c}}$, possibly $r_{\mathrm{g}} \gg c \tau_{\mathrm{c}}$, depending on $\gamma / \gamma_{\min }$ and $\epsilon_{B}$. The expansion used here should, therefore, be a good approximation away from the shock front, where $\epsilon_{B} \lesssim 10^{-2}$.

It is instructive to rewrite the above expansion parameter in terms of the ratio of fluctuating to mean quantities. In particular, using (2.5), which relates the current fluctuations to the magnetic fluctuations, one can show that, in orders of magnitude, $\delta n / n \sim k_{\max } c \delta B_{k_{\max }} /(n e c) \sim \epsilon_{B}^{1 / 2} k_{\max } c / \omega_{\mathrm{p}}$, with $\delta n$ the density of current-carrying fluctuations. Therefore, the above hierarchy $r_{\mathrm{g}} /\left(c \tau_{\mathrm{c}}\right)>1$ at $\gamma_{\min }$ also implies $\delta n<n$, i.e. small fluctuations; note that the former constraint $r_{\mathrm{g}} /\left(c \tau_{\mathrm{c}}\right)>1$ is more stringent than the latter $\delta n<n$, because $\mathscr{K} \gtrsim 10$.

Since $\delta \boldsymbol{E}_{\boldsymbol{k}^{\prime}}(\boldsymbol{\tau})$ depends solely on time, it commutes with $\mathscr{U}_{\mathscr{A}}$ (see Appendix A). Equation (2.16) can then be approximated as

$$
\begin{aligned}
& \delta f_{\alpha \boldsymbol{k}}(\boldsymbol{p}, t)=\text { init. data }- \\
& \qquad q_{\alpha} \int \mathrm{d}^{3} r \int \frac{\mathrm{d}^{3} k^{\prime}}{(2 \pi)^{3}} e^{-i \boldsymbol{k} \cdot \boldsymbol{r}+i \boldsymbol{k}^{\prime} \cdot \boldsymbol{r}} \int_{t_{0}}^{t} \mathrm{~d} \tau \boldsymbol{\delta} \boldsymbol{E}_{\boldsymbol{k}^{\prime}}(\boldsymbol{\tau}) \cdot\left\langle e^{i \boldsymbol{k}^{\prime} \cdot \boldsymbol{\Delta} \boldsymbol{r}_{\boldsymbol{s}}(\tau)} \frac{\boldsymbol{v}_{s}(\tau)}{v_{s}} \frac{\mathrm{d} \bar{f}_{\alpha}(p, \tau)}{\mathrm{d} p}\right\rangle .
\end{aligned}
$$

The quantities $\boldsymbol{r}_{\boldsymbol{s}}(\tau)$ and $\boldsymbol{v}_{\boldsymbol{s}}(\tau)$ represent the exact orbits of the particles in the fluctuating fields at time $\tau$ with boundary conditions $\boldsymbol{r}_{s}(t)=\boldsymbol{r}, \boldsymbol{v}_{\boldsymbol{s}}(t)=\boldsymbol{v}$; furthermore, $\Delta \boldsymbol{r}_{s}(\tau)=\boldsymbol{r}_{\boldsymbol{s}}(\tau)-\boldsymbol{r}$. The average over the exact orbits will be calculated further on in the limit of a magneto-static turbulence. In this limit, $v_{s}$ and $\mathrm{d} \bar{f}_{\alpha}(p) / \mathrm{d} p$ are constant in time; thus, $v_{s}=v$ in particular and these terms can be extracted from the average. Of course, dissipation is accompanied by a transfer of energy from the fields to the 
particles. This, however, takes place on a timescale $\sim \gamma_{k}^{-1}$ much larger than the scattering time of the particles, $t_{\mathrm{s}}$, so that on this latter timescale, energy flow can indeed be neglected. Furthermore, in relativistic blast waves, the turbulence energy density contains much less energy than the particles, $\epsilon_{B} \ll \epsilon_{e} \sim 0.1$ (see above references).

As a result of spatial homogeneity, the average does not depend on $\boldsymbol{r}$; it only depends on $\boldsymbol{v}$ and $t-\tau$. Therefore,

$$
\delta f_{\alpha \boldsymbol{k}}(\boldsymbol{p}, t)=\text { init. data }-q_{\alpha} \int_{t_{0}}^{t} \mathrm{~d} \tau \boldsymbol{\delta} \boldsymbol{E}_{\boldsymbol{k}}(\boldsymbol{\tau}) \cdot\left\langle e^{i \boldsymbol{k} \cdot \boldsymbol{\Delta} \boldsymbol{r}_{s}(\tau)} \boldsymbol{v}_{\boldsymbol{s}}(\tau)\right\rangle \frac{1 \mathrm{~d} \bar{f}_{\alpha}(p)}{\mathrm{d} p} .
$$

Similarly, using the fact that the above expression is written as a convolution in time, the Laplace-Fourier transform of the fluctuating part of the distribution function ends up being

$$
\delta f_{\alpha \boldsymbol{k}, \omega}(\boldsymbol{p})=\text { init. data }-q_{\alpha} \mathrm{L}_{\mathrm{L}}\left\langle e^{i \boldsymbol{k} \cdot \boldsymbol{\Delta} \boldsymbol{r}_{\boldsymbol{s}}(\tau)} \boldsymbol{v}_{\boldsymbol{s}}(\tau)\right\rangle \cdot \boldsymbol{\delta} \boldsymbol{E}_{\boldsymbol{k}, \omega} \frac{1 \mathrm{~d} \bar{f}_{\alpha}(p)}{\mathrm{d} p},
$$

with $\mathrm{L}_{\mathrm{L}}$ the Laplace transform operator; $\boldsymbol{\delta} \boldsymbol{E}_{\boldsymbol{k}, \boldsymbol{\omega}}$ represents the Fourier-Laplace transform of the fluctuating electric field. Omitting the initial data, from (2.14) and $\delta \boldsymbol{j}_{\boldsymbol{k} \omega} \equiv-i \omega \chi_{k \omega} \boldsymbol{\delta} \boldsymbol{E}_{\boldsymbol{k} \omega}$, one then extracts the nonlinear susceptibility

$$
\chi_{k \omega i j}=-\sum_{\alpha} \frac{q_{\alpha}^{2}}{-i \omega} \int \mathrm{d}^{3} p \frac{\mathrm{d} \bar{f}_{\alpha}}{\mathrm{d} p} \frac{v_{i}}{v} \mathrm{~L}_{\mathrm{L}}\left\langle e^{i \boldsymbol{k} \cdot \Delta \boldsymbol{r}_{s}(\tau)} v_{s j}(\tau)\right\rangle .
$$

One is particularly interested in the transverse susceptibility, since the turbulence is assumed magnetostatic:

$$
\chi_{k \omega, \mathrm{T}} \equiv \frac{1}{2}\left(\delta^{i j}-\frac{k^{i} k^{j}}{k^{2}}\right) \chi_{i j}
$$

\section{Analytical approximations and results}

\subsection{Nonlinear susceptibility}

In order to calculate the first-order nonlinear correction to the above damping rate, one now needs to evaluate the average over the exact orbits in (2.20). This is done in Appendix B.

Note that Appendix B assumes explicitly that the magnetic field behaves as white noise, with zero average, with a correlation time $\tau_{\mathrm{c}}$ assumed to be smaller than the scattering timescale of the particles $t_{\mathrm{s}}$. Physically, this corresponds to the transport of particles in a small-scale turbulence, i.e. to the same approximation as above, $c \tau_{\mathrm{c}}<r_{\mathrm{g}}$, with $r_{\mathrm{g}}$ the typical gyroradius of the particle defined in terms of the rms magnetic field.

The transport of particles in small-scale magneto-static turbulence is well known, see in particular Plotnikov et al. (2011) for a recent study. In this configuration, one can work out exactly the correlators that appear in (2.20), see Appendix B. One, thus, 
derives

$$
\begin{aligned}
4 \pi \chi_{k \omega, \mathrm{T}} \simeq & -\frac{1}{2} \sum_{\alpha} \frac{4 \pi q_{\alpha}^{2}}{-i \omega} 2 \pi \int \mathrm{d} p \mathrm{~d} \mu p^{2} v \frac{\mathrm{d} \bar{f}_{\alpha}}{\mathrm{d} p} \int_{0}^{+\infty} \mathrm{d} t \\
& \times \exp \left\{+i \omega t-i k v t_{\mathrm{s}} \mu\left(1-C_{1}\right)-\frac{1}{2} k^{2} v^{2} t_{\mathrm{s}}^{2} \mu^{2}\left[-\frac{1}{3}+C_{1}-C_{2}+\frac{1}{3} C_{3}\right]\right. \\
& \left.-\frac{1}{3} k^{2} v^{2} t_{\mathrm{s}}^{2}\left[\frac{t}{t_{\mathrm{s}}}-\frac{4}{3}+\frac{3}{2} C_{1}-\frac{1}{6} C_{3}\right]\right\} \\
& \times\left[\left(1+\frac{i k v \mu t_{\mathrm{s}}}{2}\right) C_{1}-i k v t_{\mathrm{s}} \mu C_{2}+\frac{i k v \mu t_{\mathrm{s}}}{2} C_{3}\right],
\end{aligned}
$$

using the short-hand notation: $C_{p} \equiv \exp \left(-p t / t_{\mathrm{s}}\right)$. The variable $\mu$ is defined as the cosine of the angle between the wave vector $\boldsymbol{k}$ and $\boldsymbol{p}$. The time integral explicits the Laplace transform over the correlation function. The above expression represents the main result of the present paper.

One notes that $k v t_{\mathrm{s}} \simeq t_{\mathrm{s}} / \tau_{\mathrm{c}}>1$, since $k \simeq 1 /\left(c \tau_{\mathrm{c}}\right)$ for a magneto-static turbulence on scale $k^{-1}$. One can, thus, approximate the above result as follows. First of all, one notes that the exponential contained in (3.1) is cut off at large times, due to the decorrelation of the particle trajectories. Introducing the following large parameter:

$$
\kappa \equiv k v t_{\mathrm{s}}
$$

which explicitly depends on particle momenta through $v$ and $t_{\mathrm{s}}$, expanding the terms in the exponential in the limit $t \ll t_{\mathrm{s}}$, one obtains (B 23), which reveals that the cut-off becomes prominent whenever $\kappa^{2} t^{3} / t_{\mathrm{s}}^{3}<1$, i.e. $t<\kappa^{-2 / 3} t_{\mathrm{s}}$. Since $\kappa>1$, this justifies the approximation of the above integral in the small-time limit $t \ll t_{\mathrm{s}}$ :

$$
\begin{aligned}
4 \pi \chi_{k \omega, \mathrm{T}} \simeq & -\frac{1}{2} \sum_{\alpha} \frac{4 \pi q_{\alpha}^{2}}{-i \omega} 2 \pi \int \mathrm{d} p \mathrm{~d} \mu p^{2} v \frac{\mathrm{d} \bar{f}_{\alpha}}{\mathrm{d} p} \\
& \times \int_{0}^{+\infty} \mathrm{d} t \exp \left[+i \omega t-i \kappa \mu t / t_{\mathrm{s}}-\frac{1}{6}\left(1-\mu^{2}\right) \kappa^{2} t^{3} / t_{\mathrm{s}}^{3}\right] \\
& \times\left[\left(1+\frac{i \kappa \mu}{2}\right) C_{1}(t)-i \kappa \mu C_{2}(t)+\frac{i \kappa \mu}{2} C_{3}(t)\right] .
\end{aligned}
$$

One can check that in the limit $t_{\mathrm{s}} \rightarrow+\infty$, one recovers the linear transverse susceptibility, as expected. This integral is of the Airy type. It can be written and further approximated by

$$
4 \pi \chi_{k \omega, \mathrm{T}}=-i \pi \sum_{\alpha} \frac{4 \pi q_{\alpha}^{2}}{\omega} \int \mathrm{d} p \mathrm{~d} \mu p^{2} v \frac{\mathrm{d} \bar{f}_{\alpha}}{\mathrm{d} p}\left[\left(1+\frac{i \kappa}{2}\right) I_{1}-i \kappa I_{2}+\frac{i \kappa}{2} I_{3}\right]\left(1-\mu^{2}\right),
$$

with

$$
\begin{aligned}
I_{p} & \equiv t_{\mathrm{s}} \int_{0}^{+\infty} \mathrm{d} \hat{t} \exp \left[i\left(\omega t_{\mathrm{s}}-\kappa \mu+i p\right) \hat{t}-\frac{1-\mu^{2}}{6} \kappa^{2} \hat{t}^{3}\right] \\
& \approx \frac{t_{\mathrm{s}}}{-i \omega t_{\mathrm{s}}+i \kappa \mu+p+\left[\frac{1-\mu^{2}}{6} \kappa^{2}\right]^{1 / 3}} .
\end{aligned}
$$

Finally, one can work out the integral over $\mu$ after dropping the slow dependence on $\left(1-\mu^{2}\right)^{1 / 3}$ in the denominators, i.e. making the substitution $\left(1-\mu^{2}\right)^{1 / 3} \sim 1$; this 
leads to

$$
\begin{aligned}
4 \pi \chi_{k \omega, \mathrm{T}} \simeq & -\pi \sum_{\alpha} \frac{4 \pi q_{\alpha}^{2}}{\omega k} \int \mathrm{d} p \mathrm{~d} \mu p^{2} v \frac{\mathrm{d} \bar{f}_{\alpha}}{\mathrm{d} p}\left\{\left(1-m_{1}\right)^{2} \ln \left(-\frac{1-m_{1}}{1+m_{1}}\right)-2 m_{1}\right. \\
& +i \kappa\left[\frac{1}{2} m_{1}\left(1-m_{1}^{2}\right) \ln \left(-\frac{1-m_{1}}{1+m_{1}}\right)-m_{2}\left(1-m_{2}^{2}\right) \ln \left(-\frac{1-m_{2}}{1+m_{2}}\right)\right. \\
& \left.\left.+\frac{1}{2} m_{3}\left(1-m_{3}\right)^{2} \ln \left(-\frac{1-m_{3}}{1+m_{3}}\right)-m_{1}^{2}+2 m_{2}^{2}-m_{3}^{2}\right]\right\}
\end{aligned}
$$

with the short-hand notation:

$$
m_{p} \equiv \frac{\omega}{k v}+i\left(p \kappa^{-1}+6^{-1 / 3} \kappa^{-2 / 3}\right) .
$$

In the limit $t_{\mathrm{s}} \rightarrow+\infty, \kappa \rightarrow+\infty$ and $m_{p} \rightarrow \omega /(k v)$; (3.6) then reduces to the standard (linear) expression for the transverse susceptibility of a relativistically hot plasma. Note that in the limit $\omega \rightarrow 0$, one can expand $\omega \chi_{k \omega, \mathrm{T}}$ to lowest order in negative powers of $\kappa$, yielding:

$$
\begin{aligned}
\left.4 \pi \omega \chi_{k \omega, \mathrm{T}}\right|_{\omega \rightarrow 0} \approx & -\pi \sum_{\alpha} \frac{4 \pi q_{\alpha}^{2}}{k} \int \mathrm{d} p \mathrm{~d} \mu p^{2} v \frac{\mathrm{d} \bar{f}_{\alpha}}{\mathrm{d} p} \\
& \times\left[i \pi-2^{5 / 3} 3^{-1 / 3} i \kappa^{-2 / 3}+i 6^{-2 / 3} \pi \kappa^{-4 / 3}+\cdots\right] .
\end{aligned}
$$

The $i \pi$ term within the brackets corresponds to the linear result; the lowest order term in $\kappa^{-2 / 3}$ indicates that the nonlinear effects tend to increase the damping rate. This will be confirmed in the full calculation below.

\subsection{Nonlinear damping rate versus $k$}

In order to evaluate the above integrals and recast them in a proper context, one needs to explicit the dependence of $\kappa$ on wavenumber and momenta; since $t_{\mathrm{s}} \propto r_{\mathrm{g}}^{2}$ and $\kappa=k v t_{\mathrm{s}}, \kappa \propto \gamma^{2}$ of course. One now assumes that the power spectrum of magnetic turbulence in Fourier space takes on a power-law shape and peaks at some maximum wavenumber $k_{\max }, \mathscr{S}_{\delta B}(k) \propto\left(k / k_{\max }\right)^{n_{B}}$, with $n_{B}>-2$ to guarantee $\tau_{\mathrm{c}} \sim 1 /\left(k_{\max } c\right)$. Linear theory predicts a damping rate $\gamma_{k} \propto k^{3}$, indicating that damping is much faster on the smaller spatial scales, as expected. Therefore, behind the shock front, the turbulent power spectrum is built up at some initial time, then gets eroded as time goes on, smaller scales being dissipated first. In short, the maximum wavenumber, at which there remains net power, becomes time-dependent. At a given time, one should, therefore, evaluate the damping rate of the turbulence at the (timedependent) maximum wavenumber $k_{\max }(t)$, since modes with smaller wavenumbers will be damped on much longer timescales. One can relate the magnetic power at time $t$ to the initial power through $\left\langle\delta B(t)^{2}\right\rangle \simeq\left\langle\delta B(0)^{2}\right\rangle\left[k_{\max }(t) / k_{\max }(0)\right]^{n_{B}+3}$. In this way, recalling that $t_{\mathrm{s}}=(3 / 2) \gamma^{2} m^{2} c^{2} /\left(\tau_{\mathrm{c}} e^{2}\left\langle\delta B^{2}\right\rangle\right)$ (see Appendix B), with $\tau_{\mathrm{c}} \simeq\left[k_{\max }(t) c\right]^{-1}$, one finds

$$
\kappa=\kappa_{0}\left[\frac{k_{\max }(t)}{k_{\max }(0)}\right]^{-n_{B}-1}\left(\frac{\gamma}{\gamma_{\min }}\right)^{2},
$$

with $\kappa_{0}$ the value of $\kappa$ at $t=0$, at $k_{\max }(0)$ and at $\gamma_{\min }$.

Interestingly, depending on the shape of the power spectrum of magnetic fluctuations, one can find situations where $\kappa$ increases or decreases as a function of $k$; in the limiting case $n_{B}=-1, \kappa$ becomes independent of the (time-dependent) maximum wavenumber behind the relativistic shock wave, meaning that $\kappa$ does not 


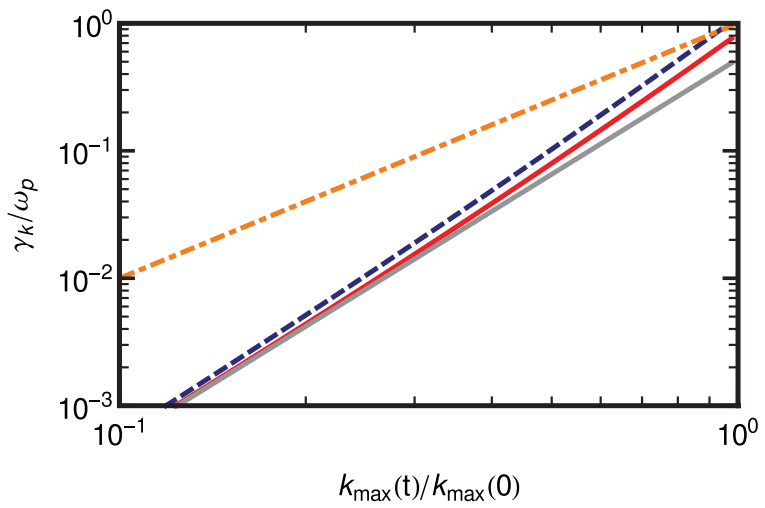

FIGURE 1. Damping rate versus wavenumber: in thick gray, the linear calculation; in thick red, the damping rate at first nonlinear order, assuming $\kappa_{0}=1$ and $n_{B}=0$, calculated through (3.1); in dashed blue, the same damping rate calculated with the approximation (3.6); in dash-dotted orange, the scattering frequency $t_{\mathrm{s}}^{-1}$ in units of $\omega_{\mathrm{p}}$ at $\gamma_{\mathrm{min}}$.

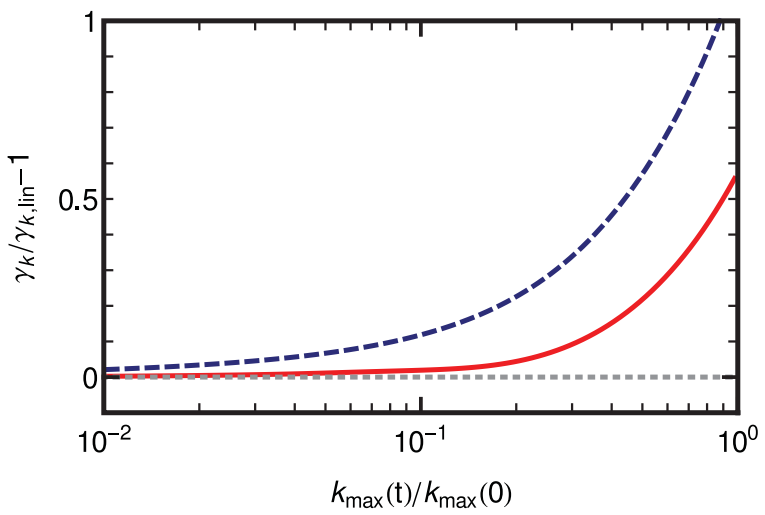

FIGURE 2. Evaluation of the nonlinear correction to the linear damping rate $\left(\gamma_{k}\right.$,lin $)$ versus wavenumber: in thick red, the correction calculated using (3.1); in dashed blue, the correction calculated with the approximation (3.6). As Fig. 1, this figure assumes $\kappa_{0}=1$ and $n_{B}=-1$.

depend on time (since injection through the shock) or, equivalently, on distance to the shock front in the downstream plasma rest frame (the shock front moving away at velocity $c / 3$ in that frame). However, if $n_{B}<-1, \kappa$ decreases with decreasing wavenumber, because erosion leaves enough power at low $k$-modes, while the effective coherence length increases, thereby leading to the eventual trapping of particles, $\kappa<1$. Since the present calculations rely on the approximation $\kappa>1$, the following assumes $n_{B}>-1$. The PIC simulations of Chang et al. (2008) further suggest that indeed $n_{B}$ is closer to zero, although this ignores the influence of high-energy particles on the turbulence, as discussed in Keshet et al. (2009) and Medvedev and Zakutnyaya (2009).

Figures 1 and 2 show a numerical evaluation of the damping rate $\gamma_{k}$, obtained through a full calculation of (3.1) and of its approximation (3.6), as a function of the time-dependent $k_{\max }$, assuming $n_{B}=0$ and $\kappa_{0}=1$. 
Figure 1 also shows the evolution of the scattering frequency $t_{\mathrm{s}}^{-1}$ versus wavenumber: this allows to verify that, at all $k_{\max }$, one has $k^{-1}<t_{\mathrm{s}}<\gamma_{k}^{-1}$, which validates the assumptions inherent to the present approach.

These figures show that the nonlinear calculation modifies the linear calculation by a factor of order unity at $k_{\max }(0)$, then converges to the linear calculation at smaller $k_{\max }$; indeed, $n_{B}=0$ implies $\kappa \propto k_{\max }^{-1}: \kappa$ increases with decreasing values of $k_{\max }$ at a same $\gamma_{\min }$, therefore the importance of nonlinear effects, which is quantified by inverse powers of $\kappa$, becomes weaker as $k_{\max }$ decreases. As mentioned above, for $n_{B}=-1$, one would find a correction at all $k_{\max }$ equal to the correction calculated at $k_{\max }(0)$.

These calculations also indicate that the nonlinear terms systematically lead to an increased damping rate, although the correction is modest. This goes contrary to the discussion in Chang et al. (2008), which conjectured that the deflection of particles by magnetic turbulence might lead to a weaker damping rate.

\section{Discussion and conclusions}

The present work studies the damping rate of the micro-turbulence which has been excited through e.g. Weibel/filamentation instabilities in the precursor of a weakly magnetized relativistic collisionless shock wave then transmitted downstream. As mentioned in Sec. 1, such calculations are directly relevant to the physics of collisionless shock waves, but also to high energy astrophysics, since the damping of the turbulence governs the strength of the magnetic field in which electrons radiate (and, therefore, the frequency at which they radiate the bulk of their energy).

In the standard afterglow model for gamma-ray burst, the canonical value for the equipartition fraction of magnetic energy density in the blast is taken as $\epsilon_{B} \sim 10^{-2}$, on the basis on afterglow observations in various wavebands, see e.g. Waxman (1997), Wijers and Galama (1999) for early determinations, and Panaitescu and Kumar (2001) for a compilation of results, which however reveals a large scatter in this parameter. Such a value would fit nicely the results of PIC simulations in the absence of dissipation, since these simulations find $\epsilon_{B} \sim 10^{-2}$ immediately downstream of the blast; the fact that $\epsilon_{B}$ remains that large up to the long time scales on which electrons can radiate gives rise to the notorious problem of the origin of these magnetic fields.

Recent detections of gamma-ray burst afterglows at high-energy $>100 \mathrm{MeV}$ may have shed a new light on this issue. If this high-energy emission indeed corresponds to the synchrotron afterglow, e.g. Kumar and Barniol Duran (2009), Ghisellini et al. (2010) and Kumar and Barniol D. (2010), these detections offer another observational constraint to pin down $\epsilon_{B}$ beyond the degeneracies inherent to most of the previous studies, see the discussion in Lemoine et al. (2013). Then one derives low values of $\epsilon_{B}$, well below the canonical one, which may be interpreted as the partial dissipation of the Weibel-generated turbulence, as described here (Lemoine 2013); in particular, assuming a power-law decay $\epsilon_{B} \propto\left(t \omega_{\mathrm{p}}\right)^{\alpha_{t}}$ as a function of comoving time, one derives $-0.5 \lesssim \alpha_{t} \lesssim-0.4$ from a handful of gamma-ray burst afterglows seen in radio, optical, X-ray and at high energy (Lemoine et al. 2013), i.e. a net dissipation. The decay of Weibel turbulence behind relativistic shock waves, thus, appears as a key ingredient in describing accurately the light curves of these extreme astronomical phenomena.

The present work presents a calculation of the damping rate to the first nonlinear order, by computing the effects of particle diffusion in the micro-turbulence. As mentioned in Sec. 1, one interest of such a calculation is to study the dependence of this correction on the power spectrum of magnetic fluctuations, which at present 
cannot be reconstructed with confidence by PIC simulations. An exact calculation is possible, thanks to the small-scale and magneto-static nature of the turbulence, which allows for an explicit calculation of the trajectory correlators which determine the amount of resonance broadening. As discussed in the main text, this work assumes that the particles are not trapped in the micro-turbulence, i.e. the scattering length is assumed larger than the coherence length of the magnetic fluctuations.

The overall influence of nonlinear terms is found to be of order unity at the maximum wavenumber, and to decrease with decreasing wavenumbers $k$, provided the three-dimensional power spectrum of magnetic fluctuations $\mathscr{S}_{\delta B} \propto k^{n_{B}}$ has an index $n_{B}>-1$. In this case, indeed, the ratio of the particle scattering timescale $t_{\mathrm{s}}$ to the coherence time $\tau_{\mathrm{c}}$ of the magnetic fluctuations increases, therefore the particle trajectories become more and more ballistic as $k$ decreases and one recovers the linear result in the small $k$ limit. The results obtained also indicate that the nonlinear correction systematically increases the damping rate.

The present results would suggest that the damping rate does follow roughly the scaling $\gamma_{k} \propto k^{3}$ predicted by linear theory, however one cannot exclude at present that the power spectrum of magnetic fluctuations is such - i.e. $n_{B}<-1-$ that effects associated to particle trapping become more and more prominent as dissipation progresses (meaning, as the time-dependent maximum wavenumber decreases). Actually, if $\gamma_{k} \propto k^{\alpha_{k}}$, one can relate the decay exponent $\alpha_{t}$ to the power spectrum index $n_{B}$ and $\alpha_{k}$ (Chang et al. 2008; Lemoine 2013):

$$
\alpha_{t}=-\frac{3+n_{B}}{\alpha_{k}} \text {. }
$$

Then, $\alpha_{k} \simeq 3$ with a value $\alpha_{t} \sim-0.5$ as suggested by observations (Lemoine et al. 2013) would imply $n_{B} \sim-1.5$, in which case the ratio $t_{\mathrm{s}} / \tau_{\mathrm{c}} \propto k^{-n_{B}-1}$ would decrease with decreasing $k$ : i.e. the nonlinear effects would become more prominent as dissipation progresses.

The present calculations cannot address the situation in which particles are effectively trapped and other theoretical tools are needed to probe this regime and to make the connection with observations. Further PIC simulations, extended in time and dimensionality, would also provide useful guidance to better characterize the scaling of $\gamma_{k}$ versus $k$. Finally, dedicated PIC simulations with an artificially set-up power spectrum might be used to probe the regime $n_{B}<-1$ in which trapping is expected to become more effective as dissipation progresses.

It is a pleasure to thank Laurent Gremillet and Guy Pelletier for very valuable suggestions and discussions. This work has been financially supported by the 'Programme National Hautes Energies' (PNHE) of the CNRS.

\section{Appendix A}

Separating the average and random parts as usual, as described in Sec. 2.2, one finds that the fluctuating part of the distribution function obeys:

$$
\left[\frac{\partial}{\partial t}+\mathscr{L}\right] f_{\alpha}(\boldsymbol{r}, \boldsymbol{p}, t)-\left\langle\delta \mathscr{L} f_{\alpha}(\boldsymbol{r}, \boldsymbol{p}, t)\right\rangle=-\delta \mathscr{L} \bar{f}_{\alpha}
$$

with:

$$
\mathscr{L}=\overline{\mathscr{L}}+\delta \mathscr{L}, \quad \overline{\mathscr{L}}=\boldsymbol{v} \cdot \frac{\partial}{\partial \boldsymbol{r}}, \quad \delta \mathscr{L}=q\left(\boldsymbol{\delta} \boldsymbol{E}+\frac{\boldsymbol{v}}{c} \times \boldsymbol{\delta} \boldsymbol{B}\right) \cdot \frac{\partial}{\partial \boldsymbol{p}}
$$


Following Weinstock (1969) and Weinstock (1970), one introduces the averaging operator $\mathscr{A}$, which takes the average over the statistical realization of the fluctuations of all quantities to its right, i.e. $\psi_{1}(\boldsymbol{r}, \boldsymbol{p}, t) \mathscr{A} \psi_{2}(\boldsymbol{r}, \boldsymbol{p}, t), \ldots, \psi_{n}(\boldsymbol{r}, \boldsymbol{p}, t)=$ $\psi_{1}\left\langle\psi_{2}, \ldots, \psi_{n}\right\rangle$. One then defines the following propagators:

$$
\begin{aligned}
\mathscr{U}: & {\left[\frac{\partial}{\partial t}+\mathscr{L}\right] \mathscr{U}\left(t, t_{0}\right)=0, \quad \mathscr{U}\left(t_{0}, t_{0}\right)=1 } \\
\mathscr{U}_{\mathscr{A}}: & {\left[\frac{\partial}{\partial t}+(1-\mathscr{A}) \mathscr{L}\right] \mathscr{U}_{\mathscr{A}}\left(t, t_{0}\right)=0, \quad \mathscr{U}_{\mathscr{A}}\left(t_{0}, t_{0}\right)=1 } \\
\overline{\mathscr{U}}: & {\left[\frac{\partial}{\partial t}+\overline{\mathscr{L}}\right] \overline{\mathscr{U}}\left(t, t_{0}\right)=-\langle\delta \mathscr{L} \mathscr{U}\rangle, \quad \overline{\mathscr{U}}\left(t_{0}, t_{0}\right)=1 . }
\end{aligned}
$$

$\mathscr{U}\left(t, t_{0}\right)$ of course represents the full propagator of the Vlasov equation; acting on a function $\psi(\boldsymbol{r}, \boldsymbol{p}, t)$, it propagates it backward in time, i.e. $\mathscr{U}\left(t, t_{0}\right) \psi(\boldsymbol{r}, \boldsymbol{p}, t)=$ $\psi\left(\boldsymbol{r}_{\boldsymbol{s}}\left(t_{0}\right), \boldsymbol{p}_{\boldsymbol{s}}\left(t_{0}\right), t_{0}\right)$ with $\boldsymbol{r}_{\boldsymbol{s}}\left(t_{0}\right), \boldsymbol{p}_{\boldsymbol{s}}\left(t_{0}\right)$ the solutions of the characteristic equations for the trajectories, such that $\boldsymbol{r}_{\boldsymbol{s}}(t)=\boldsymbol{r}$ and $\boldsymbol{p}_{\boldsymbol{s}}(t)=\boldsymbol{p}$. The merit of the propagator $\mathscr{U}_{\mathscr{A}}$ is to provide an explicit solution for $\delta f_{\alpha}(\boldsymbol{r}, \boldsymbol{p}, t)$ in terms of its initial data and the average distribution function $\bar{f}_{\alpha}$.

The various propagators $\mathscr{U}, \mathscr{U}_{\mathscr{A}}$ and $\overline{\mathscr{U}}$ are related through series expansions in powers of $\mathscr{L}$ (Birmingham and Bornatici 1971). In order to obtain a tractable expression for $\mathscr{U}_{\mathscr{A}}$, one generally truncates such series to the lowest order, which leads to $\mathscr{U}_{\mathscr{A}} \simeq \overline{\mathscr{U}}$ (Dupree 1966; Weinstock 1969, 1970). Explicitly, one finds to the next-to-leading order (Birmingham and Bornatici 1971):

$$
\mathscr{U}_{A}\left(t, t_{0}\right)=\overline{\mathscr{U}}\left(t, t_{0}\right)-\int_{t_{0}}^{t} \mathrm{~d} \tau_{1} \overline{\mathscr{U}}\left(t, \tau_{1}\right)(1-\mathscr{A}) \mathscr{L}\left(\tau_{1}\right) \bar{U}\left(\tau_{1}, t_{0}\right)+\cdots
$$

The magnitude of the next-to-leading order term relatively to the first order term is $\tau_{\mathrm{c}} e\left\langle\delta B^{2}\right\rangle^{1 / 2} /(\gamma m c)$, with $\gamma$ the Lorentz factor of the particle.

The propagator $\overline{\mathscr{U}}$ acts on a function $\psi$ by propagating it backwards in time and taking the statistical average over the exact orbits:

$$
\bar{U}\left(t, t_{0}\right) \psi(\boldsymbol{r}, \boldsymbol{p}, t)=\left\langle\psi\left[\boldsymbol{r}_{\boldsymbol{s}}\left(t_{0}\right), \boldsymbol{p}_{\boldsymbol{s}}\left(t_{0}\right), t_{0}\right]\right\rangle,
$$

with the boundary conditions $\boldsymbol{r}_{s}(t)=\boldsymbol{r}$ and $\boldsymbol{p}_{\boldsymbol{s}}(t)=\boldsymbol{p}$. Consequently, $\overline{\mathscr{U}}$ commutes with quantities that depend solely on time.

\section{Appendix B}

This Appendix calculates the correlators over the characteristic trajectories in the turbulence, which enter the expression (2.20) for the nonlinear susceptibility. All throughout this section, the index ${ }_{s}$ for the characteristic trajectories is dropped, for clarity.

The particle suffers pitch-angle scattering in a magnetostatic turbulence. As discussed in Plotnikov et al. (2011), a convenient way to calculate the transport coefficients is to write the time evolution of its velocity as a time-ordered product of an exponentiated Liouville operator:

$$
\boldsymbol{v}\left(t^{\prime}\right)=\mathscr{T} \exp \left[-\int_{t^{\prime}}^{t} \mathrm{~d} \tau_{1} \widehat{\delta \Omega}\right] \boldsymbol{v}(t)
$$


where a minus sign has been introduced in order to compute quantities at time $t^{\prime}<t$ as a function of quantities at time $t$. The rotation operator $\widehat{\delta \Omega}$ is defined as

$$
\widehat{\delta \Omega}=\delta \Omega^{a} \widehat{L}_{a}, \quad \delta \Omega^{a}=\frac{e \delta B^{a}}{\gamma m c}
$$

with $\gamma$ the Lorentz factor of the particle, $a=1,2,3, \widehat{L}_{a}$ a generator of the rotation group, with matrix components: $\widehat{L}_{a k}{ }^{l}=\epsilon_{a k}{ }^{l}\left(\epsilon_{a k l}\right.$ denotes the Levi-Civita symbol). Equation (B 1) solves the equation of motion of the particle.

The rotation operator is assumed to behave as isotropic white noise with correlation time $\tau_{\mathrm{c}}$ :

$$
\left\langle\delta \Omega^{a}\left(\tau_{1}\right)\right\rangle=0, \quad\left\langle\delta \Omega^{a}\left(\tau_{1}\right) \delta \Omega^{b}\left(\tau_{2}\right)\right\rangle=\frac{2}{3} \tau_{\mathrm{c}} \delta\left(\tau_{1}-\tau_{2}\right) \delta \Omega^{2} \delta^{a b},
$$

with $\delta \Omega^{2}=\left[e\left\langle\delta B^{2}\right\rangle^{1 / 2} /(\gamma m c)\right]^{2}$. A useful identity is: $\delta^{a b} \widehat{L}_{a} \widehat{L}_{b}=-2 \widehat{I}$, which implies $\left\langle\widehat{\delta \Omega}\left(\tau_{1}\right) \widehat{\delta \Omega}\left(\tau_{2}\right)\right\rangle=-4 \delta \Omega^{2} \tau_{\mathrm{c}} \delta\left(\tau_{1}-\tau_{2}\right) \widehat{I}$. Therefore, the average over the exact orbit gives

$$
\left\langle\boldsymbol{v}\left(t^{\prime}\right)\right\rangle=\exp \left[\frac{1}{2} \int_{t^{\prime}}^{t} \mathrm{~d} \tau_{1} \int_{t^{\prime}}^{t} \mathrm{~d} \tau_{2}\left\langle\widehat{\delta \Omega}\left(\tau_{1}\right) \widehat{\delta \Omega}\left(\tau_{2}\right)\right\rangle\right] \boldsymbol{v}=\exp \left[-\frac{2}{3} \tau_{\mathrm{c}}\left(t-t^{\prime}\right) \delta \Omega^{2}\right] \boldsymbol{v} .
$$

This correlator defines the scattering time $t_{\mathrm{s}} \equiv \frac{3}{2}\left(\tau_{\mathrm{c}} \delta \Omega^{2}\right)^{-1}$, which depends on the momentum of the particle. In the following, the generic notation $C_{a}(t) \equiv \exp \left(-a t / t_{\mathrm{s}}\right)$ is adopted, with $a$ a rational number.

The calculation of the correlator $\left\langle v_{i}\left(t_{1}\right) v_{j}\left(t_{2}\right)\right\rangle$ is more involved as it involves the product of two time-ordered exponentials. One must stress that it differs from usual velocity correlators in diffusion calculations because of the particular boundary conditions: $v_{i}(t)=v_{i}$ and $v_{j}(t)=v_{j}$. This correlator is written:

$$
v_{i}\left(t_{1}\right) v_{j}\left(t_{2}\right)=\mathscr{T} \exp \left[-\int_{t_{1}}^{t} \mathrm{~d} \tau \widehat{\delta \Omega}\right]_{i}^{k} \mathscr{T} \exp \left[-\int_{t_{2}}^{t} \mathrm{~d} \tau \widehat{\delta \Omega}\right]_{j}^{l} v_{k} v_{l}
$$

Now, if $t_{1}>t_{2}$, one rewrites

$$
\mathscr{T} \exp \left[-\int_{t_{2}}^{t} \mathrm{~d} \tau \widehat{\delta \Omega}\right]_{j}^{l}=\mathscr{T} \exp \left[-\int_{t_{1}}^{t} \mathrm{~d} \tau \widehat{\delta \Omega}\right]_{j}^{m} \mathscr{T} \exp \left[-\int_{t_{2}}^{t_{1}} \mathrm{~d} \tau \widehat{\delta \Omega}\right]_{m}^{l}
$$

and conversely if $t_{2}<t_{1}$. In the following, $\bar{t} \equiv \max \left(t_{1}, t_{2}\right)$ and $\underline{t} \equiv \min \left(t_{1}, t_{2}\right)$. Furthermore, due to the white noise nature of $\widehat{\delta \Omega}$,

$$
\begin{aligned}
&\langle\mathscr{T}\left.\exp \left[-\int_{\bar{t}}^{t} \mathrm{~d} \tau \widehat{\delta \Omega}\right] \mathscr{T} \exp \left[-\int_{\bar{t}}^{t} \mathrm{~d} \tau \widehat{\delta \Omega}\right] \mathscr{T} \exp \left[-\int_{\underline{t}}^{\bar{t}} \mathrm{~d} \tau \widehat{\delta \Omega}\right]\right\rangle \\
&=\left\langle\mathscr{T} \exp \left[-\int_{\bar{t}}^{t} \mathrm{~d} \tau \widehat{\delta \Omega}\right] \mathscr{T} \exp \left[-\int_{\bar{t}}^{t} \mathrm{~d} \tau \widehat{\delta \Omega}\right]\right\rangle\left\langle\mathscr{T} \exp \left[-\int_{\underline{t}}^{\bar{t}} \mathrm{~d} \tau \widehat{\delta \Omega}\right]\right\rangle .
\end{aligned}
$$

Finally, the action of $\left\langle\mathscr{T} \exp \left[-\int_{\underline{t}}^{\bar{t}} \mathrm{~d} \tau \widehat{\delta \Omega}\right]\right\rangle$ on $v_{k} v_{l}$ gives a factor $C_{1}(\bar{t}-\underline{t}) v_{k} v_{l}$, see (B 4). One, therefore, needs to calculate only the first average on the r.h.s. of (B 7).

Here, a key observation is to note that the product of those two time ordered exponentials can be rewritten as the time ordered exponential of a tensorial operator; 
this is demonstrated in Appendix C. Expliciting the matrix components:

$$
\mathscr{T} \exp \left[-\int_{\bar{t}}^{t} \mathrm{~d} \tau \widehat{\delta \Omega}\right]_{i}{ }^{k} \mathscr{T} \exp \left[-\int_{\bar{t}}^{t} \mathrm{~d} \tau \widehat{\delta \Omega}\right]_{j}{ }^{l}=\mathscr{T} \exp \left[-\int_{\bar{t}}^{t} \mathrm{~d} \tau \delta W\right]_{i j}{ }^{k l},
$$

with

$$
\delta W_{i j}{ }^{k l}=\delta W^{<_{i j}{ }^{k l}+\delta W^{>}{ }_{i j}{ }^{k l}, \quad \delta W^{<_{i j}{ }^{k l}}=\delta \Omega_{i}^{k} \delta_{j}^{l}, \quad \delta W^{<}{ }_{i j}{ }^{k l}=\delta_{i}^{k} \delta \Omega_{j}^{l} .}
$$

The tensor product rule in the time ordered exponential is understood as:

$$
\delta W \cdot \delta W_{i j}{ }^{k l} \equiv \delta W_{i j}{ }^{m n} \delta W_{m n}{ }^{k l} .
$$

One, therefore, obtains:

$$
\left\langle v_{i}\left(t_{1}\right) v_{j}\left(t_{2}\right)\right\rangle=\mathscr{T} \exp \left[\frac{1}{2} \int_{\bar{t}}^{t} \int_{\bar{t}}^{t} \mathrm{~d} \tau_{1} \mathrm{~d} \tau_{2}\left\langle\delta W\left(\tau_{1}\right) \cdot \delta W\left(\tau_{2}\right)\right\rangle\right]_{i j}{ }_{i j} C_{1}(\bar{t}-\underline{t}) v_{k} v_{l} .
$$

Now, using the identity

$$
\begin{aligned}
\left\langle\delta \Omega_{i}{ }^{m}\left(\tau_{1}\right) \delta \Omega_{j}{ }^{n}\left(\tau_{2}\right)\right\rangle & =\left\langle\delta \Omega^{a}\left(\tau_{1}\right) \delta \Omega^{b}\left(\tau_{2}\right)\right\rangle{\widehat{L_{a i}}}^{m}{\widehat{L_{b j}}}^{n} \\
& =\frac{2}{3} \tau_{c} \delta\left(\tau_{1}-\tau_{2}\right) \delta \Omega^{2}\left[\delta_{i j} \delta^{m n}-\delta_{i}{ }^{m} \delta_{j}{ }^{n}\right]
\end{aligned}
$$

one finds

$$
\left\langle\delta W\left(\tau_{1}\right) \cdot \delta W\left(\tau_{2}\right)\right\rangle_{i j}{ }^{k l}=\frac{2}{3} \tau_{\mathrm{c}} \delta\left(\tau_{1}-\tau_{2}\right) \delta \Omega^{2}\left[-4 \delta_{i}{ }^{k} \delta_{j}{ }^{l}-2 \delta_{i}{ }^{l} \delta_{j}{ }^{k}+2 \delta_{i j} \delta^{k l}\right] .
$$

This operator eventually acts on $v_{k} v_{l}$, which is symmetric in $k$ and $l$, therefore one can keep only the symmetric part. Define, therefore,

$$
M_{i j}{ }^{k l}=\delta_{i}{ }^{k} \delta_{j}{ }^{l}+\delta_{i}{ }^{l} \delta_{j}{ }^{k}-\frac{2}{3} \delta_{i j} \delta^{k l}
$$

in terms of which one rewrites the symmetrized average, as indicated by the symbol $(k l)$ :

$$
\left\langle\delta W\left(\tau_{1}\right) \cdot \delta W\left(\tau_{2}\right)\right\rangle_{i j}{ }^{(k l)}=-2 \tau_{\mathrm{c}} \delta\left(\tau_{1}-\tau_{2}\right) \delta \Omega^{2} M_{i j}{ }^{k l} .
$$

Finally, the $M$ operator satisfies: $M \cdot M=2 M$ so that

$$
\exp (\alpha M)=1-\frac{M}{2}+e^{2 \alpha} \frac{M}{2},
$$

and

$$
M_{i j}{ }^{k l} v_{k} v_{l}=2 v_{i} v_{j}-\frac{2}{3} v^{2} \delta_{i j}
$$

Combining together the above results, one ends up with:

$$
\left\langle v_{i}\left(t_{1}\right) v_{j}\left(t_{2}\right)\right\rangle=C_{1}(\bar{t}-\underline{t})\left\{C_{3}(t-\bar{t}) v_{i} v_{j}+\frac{v^{2}}{3} \delta_{i j}\left[1-C_{3}(t-\bar{t})\right]\right\},
$$

which has a simple interpretation: the correlator vanishes for time intervals $\bar{t}-\underline{t}$ larger than $t_{\mathrm{s}}$, else it tends towards $v_{i} v_{j}$ (the boundary conditions at time $t$ ) if $t-\bar{t} \ll t_{\mathrm{s}} / 3$, or to the isotropic average $\delta_{i j} v^{2} / 3$ in the opposite limit. 
One then derives easily the position correlator, with $\Delta r_{i}\left(t^{\prime}\right) \equiv r_{i}\left(t^{\prime}\right)-r_{i}$, including the boundary condition $r_{i}(t)=r_{i}$ :

$$
\begin{aligned}
\left\langle\Delta r_{i}\left(t^{\prime}\right) \Delta r_{j}\left(t^{\prime}\right)\right\rangle= & \int_{t^{\prime}}^{t} \int_{t^{\prime}}^{t} \mathrm{~d} \tau_{1} \mathrm{~d} \tau_{2}\left\langle v_{i}\left(\tau_{1}\right) v_{j}\left(\tau_{2}\right)\right\rangle \\
= & 2 t_{\mathrm{s}}^{2}\left\{\left[\frac{1}{3}+\frac{1}{6} C_{3}\left(t-t^{\prime}\right)-\frac{1}{2} C_{1}\left(t-t^{\prime}\right)\right] v_{i} v_{j}+\right. \\
& \left.\times\left[\frac{t-t^{\prime}}{t_{\mathrm{s}}}-\frac{4}{3}+\frac{3}{2} C_{1}\left(t-t^{\prime}\right)-\frac{1}{6} C_{3}\left(t-t^{\prime}\right)\right] \frac{v^{2}}{3} \delta_{i j}\right\} .
\end{aligned}
$$

Similarly, one obtains

$$
\left\langle\Delta r_{i}\left(t^{\prime}\right)\right\rangle=-t_{\mathrm{s}}\left[1-C_{1}\left(t-t^{\prime}\right)\right] v_{i}
$$

hence

$$
\begin{aligned}
&\left\langle\Delta r_{i}\left(t^{\prime}\right) \Delta r_{j}\left(t^{\prime}\right)\right\rangle-\left\langle\Delta r_{i}\left(t^{\prime}\right)\right\rangle\left\langle\Delta r_{j}\left(t^{\prime}\right)\right\rangle \\
&= t_{\mathrm{s}}^{2}\left\{\left[-\frac{1}{3}+C_{1}\left(t-t^{\prime}\right)-C_{2}\left(t-t^{\prime}\right)+\frac{1}{3} C_{3}\left(t-t^{\prime}\right)\right] v_{i} v_{j}\right. \\
&\left.\quad+\frac{2}{3} v^{2} \delta_{i j}\left[\frac{t-t^{\prime}}{t_{\mathrm{s}}}-\frac{4}{3}+\frac{3}{2} C_{1}\left(t-t^{\prime}\right)-\frac{1}{6} C_{3}\left(t-t^{\prime}\right)\right]\right\} .
\end{aligned}
$$

The average $\left\langle\exp \left[i \boldsymbol{k} \cdot \boldsymbol{\Delta} \boldsymbol{r}\left(t^{\prime}\right)\right]\right\rangle$ can be truncated at the first cumulant, leading to

$$
\left\langle\exp \left[i \boldsymbol{k} \cdot \boldsymbol{\Delta} \boldsymbol{r}\left(t^{\prime}\right)\right]\right\rangle \simeq \exp \left\{i k^{i} \cdot\left\langle\Delta r_{i}\left(t^{\prime}\right)\right\rangle-\frac{1}{2} k_{i} k_{j}\left[\left\langle\Delta r_{i} \Delta r_{j}\right\rangle-\left\langle\Delta r_{i}\right\rangle\left\langle\Delta r_{j}\right\rangle\right]\right\}
$$

In particular, the small-time limit $t-t^{\prime} \ll t_{\mathrm{s}}$ will be useful:

$$
\left\langle\exp \left[i \boldsymbol{k} \cdot \boldsymbol{\Delta r}\left(t^{\prime}\right)\right]\right\rangle \simeq \exp \left[-i k v \mu\left(t-t^{\prime}\right)-\frac{1}{6} k^{2} v^{2}\left(1-\mu^{2}\right)\left(t-t^{\prime}\right)^{3} / t_{\mathrm{s}}\right],
$$

with $\mu=\boldsymbol{k} \boldsymbol{v} /(k v)$.

The correlator which enters the expression for the nonlinear susceptibility is

$$
\left\langle\exp \left[i \boldsymbol{k} \cdot \boldsymbol{\Delta} \boldsymbol{r}\left(t^{\prime}\right)\right] v_{j}\left(t^{\prime}\right)\right\rangle=\left\langle\exp \left[i \boldsymbol{k} \cdot \boldsymbol{\Delta} \boldsymbol{r}\left(t^{\prime}\right)+\boldsymbol{\Delta} \boldsymbol{v} \cdot \partial / \partial \boldsymbol{v}\right]\right\rangle v_{j}
$$

It is understood that $\boldsymbol{\Delta} \boldsymbol{v}=\boldsymbol{v}\left(t^{\prime}\right)-\boldsymbol{v}$ and the partial derivative $\partial / \partial \boldsymbol{v}$ does not act on $\boldsymbol{\Delta} \boldsymbol{v}$. As before, the average of the exponential can be truncated at the first cumulant, leading to

$$
\begin{aligned}
& \left\langle\exp \left[i \boldsymbol{k} \cdot \boldsymbol{\Delta} \boldsymbol{r}\left(t^{\prime}\right)\right] v_{j}\left(t^{\prime}\right)\right\rangle \\
& =\exp \left\{i k^{i} \cdot\left\langle\Delta r_{i}\left(t^{\prime}\right)\right\rangle-\frac{1}{2} k_{i} k_{j}\left[\left\langle\Delta r_{i} \Delta r_{j}\right\rangle-\left\langle\Delta r_{i}\right\rangle\left\langle\Delta r_{j}\right\rangle\right]\right. \\
& \left.\quad+i k_{i}\left\langle\Delta r_{i}\left(t^{\prime}\right) \Delta v_{k}\left(t^{\prime}\right)\right\rangle \partial / \partial v_{k}-i k_{i}\left\langle\Delta r_{i}\left(t^{\prime}\right)\right\rangle\left\langle\Delta v_{k}\left(t^{\prime}\right)\right\rangle \partial / \partial v_{k}\right\} v_{j},
\end{aligned}
$$

where the second-order cumulant $\left\langle\Delta v_{j} \Delta v_{k}\right\rangle \partial / \partial v_{j} \partial / \partial v_{k}$ has not been considered because it vanishes when acting on $\boldsymbol{v}$. Expanding the exponential in $\partial / \partial v_{j}$ to first 
order, one ends up with

$$
\begin{aligned}
\left\langle\exp \left[i \boldsymbol{k} \cdot \boldsymbol{\Delta} \boldsymbol{r}\left(t^{\prime}\right)\right] v_{j}\left(t^{\prime}\right)\right\rangle=\langle & \left.\exp \left[i \boldsymbol{k} \cdot \boldsymbol{\Delta} \boldsymbol{r}\left(t^{\prime}\right)\right]\right\rangle \\
& \times\left\{\left\langle v_{j}\right\rangle+i k_{i}\left[\left\langle\Delta r_{i}\left(t^{\prime}\right) \Delta v_{j}\left(t^{\prime}\right)\right\rangle-\left\langle\Delta r_{i}\left(t^{\prime}\right)\right\rangle\left\langle\Delta v_{j}\left(t^{\prime}\right)\right\rangle\right]\right\} \\
= & \left.\exp \left[i \boldsymbol{k} \cdot \boldsymbol{\Delta} \boldsymbol{r}\left(t^{\prime}\right)\right]\right\rangle\left\{C_{1}\left(t-t^{\prime}\right) v_{j}\right. \\
& +i k v t_{\mathrm{s}} \mu\left[\frac{1}{2} C_{1}\left(t-t^{\prime}\right)-C_{2}\left(t-t^{\prime}\right)+\frac{1}{2} C_{3}\left(t-t^{\prime}\right)\right] v_{j} \\
& \left.-\frac{i}{3} k_{j} v^{2} t_{\mathrm{s}}\left[1-\frac{3}{2} C_{1}\left(t-t^{\prime}\right)+\frac{1}{2} C_{3}\left(t-t^{\prime}\right)\right]\right\} .
\end{aligned}
$$

In calculating the transverse susceptibility, the (longitudinal) last term $\propto k_{j}$ disappears, of course.

\section{Appendix C}

In order to demonstrate (B 8), one needs to expand the time ordered exponentials. The left hand side reads

$$
\begin{aligned}
& \mathscr{T} \exp \left[-\int_{\bar{t}}^{t} \mathrm{~d} \tau \widehat{\delta \Omega}\right]_{i}^{k} \mathscr{T} \exp \left[-\int_{\bar{t}}^{t} \mathrm{~d} \tau \widehat{\delta \Omega}\right]_{j}^{l} \\
&=\sum_{m, n=0}^{+\infty} \frac{1}{m ! n !} \overbrace{\int_{\bar{t}}^{t} \mathrm{~d} \tau_{1} \delta \Omega_{i_{1}}^{i_{2}} \int_{\bar{t}}^{\tau_{1}} \mathrm{~d} \tau_{2} \delta \Omega_{i_{2}}^{i_{3}} \ldots}^{m \text { arguments }} \overbrace{\int_{\bar{t}}^{t} \mathrm{~d} \tau_{1}^{\prime} \delta \Omega_{j_{1}}^{j_{2}} \int_{\bar{t}}^{\tau_{1}^{\prime}} \mathrm{d} \tau_{2}^{\prime} \delta \Omega_{j_{2}}^{j_{3}} \ldots}^{n \text { arguments }}
\end{aligned}
$$

and it is understood that $i_{1}=i, i_{m+1}=k, j_{1}=j, j_{n+1}=l$. The product of the $m$ by $n$ integrals can be written as a single time ordered sequence as follows. For the sake of clarity, one first rewrites $\delta \Omega^{<}$the operators with $i$ indices and $\delta \Omega^{>}$the operators with $j$ indices and one keeps in mind that all $\delta \Omega^{<}$operators are contracted one with the other according to the time ordered sequence, and similarly for the $\delta \Omega^{>}$ operators. Then, one breaks the integral $\int_{t}^{t} \mathrm{~d} \tau_{1}^{\prime} \delta \Omega_{j_{1}}^{>j_{2}}$ over the time intervals $\left[t, \tau_{m}\right]$, $\left[\tau_{m}, \tau_{m-1}\right], \ldots,\left[\tau_{1}, t\right]$ and one reorders the sequence, noting that (indices discarded):

$$
\int_{\bar{t}}^{\tau_{i}} \mathrm{~d} \tau_{i+1} \delta \Omega^{<} \int_{\tau_{i+1}}^{\tau_{i}} \mathrm{~d} \tau_{j} \delta \Omega^{>}=\int_{\bar{t}}^{\tau_{i}} \mathrm{~d} \tau_{j} \delta \Omega^{>} \int_{\bar{t}}^{\tau_{j}} \mathrm{~d} \tau_{i+1} \delta \Omega^{<} .
$$

Repeating this exercise for all $\delta \Omega^{>}$integrals, in the order of the time sequence, one ends up with a time ordered sum over all possible permutations $\sigma_{m n}$ of the operators:

$$
\begin{aligned}
\mathscr{T} & \exp \left[-\int_{\bar{t}}^{t} \mathrm{~d} \tau \widehat{\delta \Omega}\right]_{i}^{k} \mathscr{T} \exp \left[-\int_{\bar{t}}^{t} \mathrm{~d} \tau \widehat{\delta \Omega}\right]_{j}^{l} \\
= & \sum_{m, n=0}^{+\infty} \frac{1}{m ! n !} \sum_{\sigma_{m n}} \int_{\bar{t}}^{t} \mathrm{~d} \tau_{1} \delta \Omega^{\sigma_{m n}(1)} \int_{\bar{t}}^{\tau_{1}} \mathrm{~d} \tau_{2} \delta \Omega^{\sigma_{m n}(2)} \ldots \int_{\bar{t}}^{\tau_{m+n}} \mathrm{~d} \tau_{m+n} \delta \Omega^{\sigma_{m n}(m+n)} .
\end{aligned}
$$


The permutation is defined by: $\sigma_{m n}(a)=(>$ or $<$ ), with $m$ copies of $<$ and $n$ copies of $>$. The indices have been discarded, but it is understood that all operators are contracted within their respective $<$ or $>$ families, as mentioned previously. One then notes that this contraction sequence can be rewritten as the tensor product of $\delta W^{<}, \delta W^{>}$operators introduced above, namely:

$$
\left[\delta \Omega^{\sigma_{m n}(1)} \delta \Omega^{\sigma_{m n}(2)} \ldots \delta \Omega^{\sigma_{m n}(m+n)}\right]_{i j}{ }^{k l}=\left[\delta W^{\sigma_{m n}(1)} \cdot \delta W^{\sigma_{m n}(2)} \cdot \ldots \cdot \delta W^{\sigma_{m n}(m+n)}\right]_{i j}{ }^{k l}
$$

since $\delta W^{<}$acts non-trivially only on $i$-type indices, while $\delta W^{>}$acts non-trivially only on $j$-type indices.

Finally, one uses:

$$
\begin{aligned}
& \sum_{m, n=0}^{+\infty} \frac{1}{m ! n !} \mathscr{T} \int \mathrm{d} \tau_{1} \delta W^{\sigma_{m n}(1)} \int \mathrm{d} \tau_{2} \delta W^{\sigma_{m n}(2)} \ldots \\
& \quad=\sum_{p=0}^{\infty} \frac{1}{p !} \sum_{m=0}^{p} \frac{p !}{m !(p-m) !} \mathscr{T} \int \mathrm{d} \tau_{1} \delta W^{\sigma_{m, p-m}(1)} \int \mathrm{d} \tau_{2} \delta W^{\sigma_{m, p-m}(2)} \ldots
\end{aligned}
$$

to obtain

$$
\mathscr{T} \exp \left[-\int_{\bar{t}}^{t} \mathrm{~d} \tau \widehat{\delta \Omega}\right]_{i}^{k} \mathscr{T} \exp \left[-\int_{\bar{t}}^{t} \mathrm{~d} \tau \widehat{\delta \Omega}\right]_{j}^{l}=\sum_{p=0}^{\infty} \frac{1}{p !} \mathscr{T}\left[\int_{\underline{t}}^{\bar{t}} \mathrm{~d} \tau\left(\delta W^{<}+\delta W^{>}\right)\right]_{i j}^{p l}
$$

which gives the desired result.

\section{REFERENCES}

Achterberg, A., Gallant, Y. A., Kirk, J. G. and Guthmann, A. W. 2001 Particle acceleration by ultrarelativistic shocks: theory and simulations. Month. Not. Roy. Astron. Soc. 328, 393-408.

Achterberg, A. and Wiersma, J. 2007 The Weibel instability in relativistic plasmas. I. Linear theory. Astron. Astrophys. 475, 1-18.

Achterberg, A., Wiersma, J. and Norman, C. A. 2007 The Weibel instability in relativistic plasmas. II. Nonlinear theory and stabilization mechanism. Astron. Astrophys. 475, 19-36.

Bednarz, J. and Ostrowski, M. 1998 Energy spectra of cosmic rays accelerated at ultrarelativistic shock waves. Phys. Rev. Lett. 80, 3911-3914.

Ben-Israel, I., Piran, T., Eviatar, A. and Weinstock, J. 1975 A statistical theory of electromagnetic waves in turbulent plasmas. Astrophys. Space Sci. 38, 125-155.

Bergman, J. and Eliasson, B. 2001 Linear wave dispersion laws in unmagnetized relativistic plasma: analytical and numerical results. Phys. Plasmas 8, 1482-1492.

Bezzerides, B. and Weinstock, J. 1972 Nonlinear saturation of parametric instabilities. Phys. Rev. Lett. 28, 481-484.

Birmingham, T. J. and Bornatici, M. 1971 Propagators in strong plasma turbulence. Phys. Fluids 14, 2239-2241.

Blandford, R. and Eichler, D. 1987 Particle acceleration at astrophysical shocks: a theory of cosmic ray origin. Phys. Rep. 154, 1-75.

Blandford, R. D. and McKee, C. F. 1976 Fluid dynamics of relativistic blast waves. Phys. Fluids 19, $1130-1138$.

Bret, A., Gremillet, L. and Dieckmann, M. E. 2010 Multidimensional electron beam-plasma instabilities in the relativistic regime. Phys. Plasmas 17(12), 120501.

Chang, P., Spitkovsky, A. and Arons, J. 2008 Long-term evolution of magnetic turbulence in relativistic collisionless shocks: electron-positron plasmas. Astrophys. J. 674, 378-387.

Drake, R. P. and Gregori, G. 2012 Design considerations for unmagnetized collisionless-shock measurements in homologous flows. Astrophys. J. 749, 171. 
Dum, C. T. and Dupree, T. H. 1970 Nonlinear stabilization of high-frequency instabilities in a magnetic field. Phys. Fluids 13, 2064-2081.

Dupree, T. H. 1966 A perturbation theory for strong plasma turbulence. Phys. Fluids 9, 1773-1782.

Felten, T. and Schlickeiser, R. 2013a Spontaneous electromagnetic fluctuations in unmagnetized plasmas. V. Relativistic form factors of weakly damped/amplified thermal modes. Phys. Plasmas 20 (8), 082117.

Felten, T. and Schlickeiser, R. 2013b Spontaneous electromagnetic fluctuations in unmagnetized plasmas. VI. Transverse, collective mode for arbitrary distribution functions. Phys. Plasmas 20 (10), 104502.

Felten, T., Schlickeiser, R., Yoon, P. H. and Lazar, M. 2013 Spontaneous electromagnetic fluctuations in unmagnetized plasmas. II. Relativistic form factors of aperiodic thermal modes. Phys. Plasmas 20(5), 052113.

Ghisellini, G., Ghirlanda, G., Nava, L. and Celotti, A. $2010 \mathrm{GeV}$ emission from gamma-ray bursts: a radiative fireball? Month. Not. Roy. Astron. Soc. 403, 926-937.

Gruzinov, A. 2000 Ultra-relativistic blast wave: stability and strong non-universality. ArXiv Astrophys. e-prints .

Gruzinov, A. and Waxman, E. 1999 Gamma-ray burst afterglow: polarization and analytic light curves. Astrophys. J. 511, 852-861.

Kato, T. N. and Takabe, H. 2008 Nonrelativistic collisionless shocks in unmagnetized electron-ion plasmas. Astrophys. J. Lett. 681, L93-L96.

Katz, B., Keshet, U. and Waxman, E. 2007 Self-similar collisionless shocks. Astrophys. J. 655, 375-390.

Kelner, S. R., Aharonian, F. A. and Khangulyan, D. 2013 On the jitter radiation. Astrophys. J. 774, 61.

Keshet, U., Katz, B., Spitkovsky, A. and Waxman, E. 2009 Magnetic field evolution in relativistic unmagnetized collisionless shocks. Astrophys. J. Lett. 693, L127-L130.

Keshet, U. and Waxman, E. 2005 Energy spectrum of particles accelerated in relativistic collisionless shocks. Phys. Rev. Lett. 94(11), 111102.

Kirk, J. G., Guthmann, A. W., Gallant, Y. A. and Achterberg, A. 2000 Particle acceleration at ultrarelativistic shocks: an eigenfunction method. Astrophys. J. 542, 235-242.

Kocharovsky, V. V., Kocharovsky, V. V. and Martyanov, V. J. 2010 Self-consistent current sheets and filaments in relativistic collisionless plasma with arbitrary energy distribution of particles. Phys. Rev. Lett. 104(21), 215002.

Kumar, P. and Barniol D., R. 2009 On the generation of high-energy photons detected by the Fermi Satellite from gamma-ray bursts. Month. Not. Roy. Astron. Soc. 400, L75-L79.

Kumar, P. and Barniol D., R. 2010 External forward shock origin of high-energy emission for three gamma-ray bursts detected by Fermi. Month. Not. Roy. Astron. Soc. 409, 226-236.

Lemoine, M. 2013 Synchrotron signature of a relativistic blast wave with decaying microturbulence. Month. Not. Roy. Astron. Soc. 428, 845-866.

Lemoine, M., Li, Z. and Wang, X.-Y. 2013 On the magnetization of gamma-ray burst blast waves. Month. Not. Roy. Astron. Soc. 435, 3009-3016.

Lemoine, M. and Pelletier, G. 2003 Particle transport in tangled magnetic fields and Fermi acceleration at relativistic shocks. Astrophys. J. 589, L73-L76.

Lemoine, M. and Pelletier, G. 2010 On electromagnetic instabilities at ultra-relativistic shock waves. Month. Not. Roy. Astron. Soc. 402, 321-334.

Lemoine, M. and Pelletier, G. 2011 Dispersion and thermal effects on electromagnetic instabilities in the precursor of relativistic shocks. Month. Not. Roy. Astron. Soc. 417, 1148-1161.

Lemoine, M., Pelletier, G. and Revenu, B. 2006 On the efficiency of Fermi acceleration at relativistic shocks. Astrophys. J. Lett. 645, L129-L132.

Levinson, A. 2009 Convective instability of a relativistic ejecta decelerated by a surrounding medium: an origin of magnetic fields in gamma-ray bursts? Astrophys. J. Lett. 705, L213-L216.

Levinson, A. 2010 Relativistic rayleigh-taylor instability of a decelerating shell and its implications for gamma-ray bursts. Geophys. Astrophys. Fluid Dyn. 104, 85-111.

Lyubarsky, Y. and Eichler, D. 2006 Are gamma-ray burst shocks mediated by the weibel instability? Astrophys. J. 647, 1250-1254. 
Mart'yanov, V. Y., Kocharovsky, V. V. and Kocharovsky, V. V. 2008 Saturation of relativistic Weibel instability and the formation of stationary current sheets in collisionless plasma. Sov. J. Exp. Theor. Phys. 107, 1049-1060.

Medvedev, M. V., Fiore, M., Fonseca, R. A., Silva, L. O. and Mori, W. B. 2005 Long-time evolution of magnetic fields in relativistic Gamma-Ray burst shocks. Astrophys. J. 618, L75-L78.

Medvedev, M. V., Frederiksen, J. T., Haugbølle, T. and Nordlund, A. A. 2011 Radiation signatures of sub-larmor scale magnetic fields. Astrophys. J. 737, 55.

Medvedev, M. V. and Loeb, A. 1999 Generation of magnetic fields in the relativistic shock of Gamma-Ray burst sources. Astrophys. J. 526, 697-706.

Medvedev, M. V. and Zakutnyaya, O. V. 2009 Magnetic fields and cosmic rays in GRBs: a self-similar collisionless foreshock. Astrophys. J. 696, 2269-2274.

Mizuno, Y., Pohl, M., Niemiec, J., Zhang, B., Nishikawa, K.-I. and Hardee, P. E. 2014 Magnetic field amplification and saturation in turbulence behind a relativistic shock. Month. Not. Roy. Astron. Soc. 439, 3490-3503.

Moiseev, S. S. and Sagdeev, R. Z. 1963 Collisionless shock waves in a plasma in a weak magnetic field. J. Nucl. Energy 5, 43-47.

Niemiec, J., Ostrowski, M. and Pohl, M. 2006 Cosmic-ray acceleration at ultrarelativistic shock waves: effects of downstream short-wave Turbulence. Astrophys. J. 650, 1020-1027.

Panaitescu, A. and Kumar, P. 2001 Fundamental physical parameters of collimated Gamma-Ray burst afterglows. Astrophys. J. Lett. 560, L49-L53.

Pelletier, G. 1977 Renormalization method and singularities in the theory of Langmuir turbulence. J. Plasma Phys. 18, 49-76.

Pelletier, G., Lemoine, M. and Marcowith, A. 2009 On Fermi acceleration and magnetohydrodynamic instabilities at ultra-relativistic magnetized shock waves. Month. Not. Roy. Astron. Soc. 393, 587-597.

Piran, T. 2004 The physics of gamma-ray bursts. Rev. Mod. Phys. 76, 1143-1210.

Plotnikov, I., Pelletier, G. and Lemoine, M. 2011 Particle transport in intense small-scale magnetic turbulence with a mean field. Astron. Astrophys. 532, A68.

Pokhotelov, O. A. and Amariutei, O. A. 2011 Quasi-linear dynamics of Weibel instability. Ann. Geophys. 29, 1997-2001.

Rabinak, I., Katz, B. and Waxman, E. 2011 Long-wavelength unstable modes in the far upstream of relativistic collisionless shocks. Astrophys. J. 736, 157.

Ruyer, C., Gremillet, L., Bénisti, D. and Bonnaud, G. 2013 Electromagnetic fluctuations and normal modes of a drifting relativistic plasma. Phys. Plasmas 20(11), 112104.

Shaisultanov, R., Lyubarsky, Y. and Eichler, D. 2012 Stream instabilities in relativistically hot plasma. Astrophys. J. 744, 182.

Sironi, L. and Goodman, J. 2007 Production of magnetic energy by macroscopic turbulence in GRB afterglows. Astrophys. J. 671, 1858-1867.

Sironi, L. and Spitkovsky, A. 2009 Synthetic Spectra from particle-in-cell simulations of relativistic collisionless shocks. Astrophys. J. Lett. 707, L92-L96.

Sironi, L., Spitkovsky, A. and Arons, J. 2013 The maximum energy of accelerated particles in relativistic collisionless shocks. Astrophys. J. 771, 54.

Spitkovsky, A. 2008a On the structure of relativistic collisionless shocks in electron-Ion plasmas. Astrophys. J. Lett. 673, L39-L42.

Spitkovsky, A. 2008b Particle acceleration in relativistic collisionless shocks: Fermi process at last? Astrophys. J. Lett. 682, L5-L8.

Waxman, E. 1997 Gamma-Ray-burst afterglow: supporting the cosmological fireball model, constraining parameters, and making predictions. Astrophys. J. Lett. 485, L5-L8.

Weinstock, J. 1969 Formulation of a statistical theory of strong plasma turbulence. Phys. Fluids 12, $1045-1058$.

Weinstock, J. 1970 Turbulent plasmas in a magnetic field-a statistical theory. Phys. Fluids 13, $2308-2316$.

Weinstock, J. 1972 Nonlinear theory of frequency shifts and broadening of plasma waves. Phys. Fluids 15, 454-459.

Weinstock, J. and Bezzerides, B. 1973 Nonlinear saturation of parametric instabilities: spectrum of turbulence and enhanced collision frequency. Phys. Fluids 16, 2287-2303. 
Wiersma, J. and Achterberg, A. 2004 Magnetic field generation in relativistic shocks. An early end of the exponential Weibel instability in electron-proton plasmas. Astron. Astrophys. 428, 365-371.

Wijers, R. A. M. J. and Galama, T. J. 1999 Physical parameters of GRB 970508 and GRB 971214 from their afterglow synchrotron emission. Astrophys. J. 523, 177-186.

Yoon, P. H., Schlickeiser, R. and Kolberg, U. 2014 Thermal fluctuation levels of magnetic and electric fields in unmagnetized plasma: the rigorous relativistic kinetic theory. Phys. Plasmas 21(3), 032109. 